\title{
Volatilidad de tipo de cambio y dolarización de empresas bancarias del sistema financiero peruano, 2012 - 2018
}

\author{
Volatility of the exchange rate and the dollarization of the \\ banking companies of the peruvian financial system, \\ $2012-2018$
}

\begin{abstract}
RESUMEN
La dolarización de las empresas bancarias del sistema financiero tiene fuertes implicancias en el desarrollo y toma de decisiones de estas al generarse los "descalces" de monedas y de plazos, incidiendo en la administración, planificación, gestión de costos, riesgos entre otros de la misma, que finalmente afecta a los resultados económicos financieros de dichas empresas. Existen muchas causas que explican la dolarización en las empresas bancarias. Acorde a lo visto en muchos países principalmente en vías de desarrollo y de economías pequeñas, la volatilidad del tipo de cambio ha jugado un rol importante en el comportamiento de la dolarización. Los principales resultados del estudio son: la volatilidad del tipo de cambio influye significativamente en la dolarización de las empresas bancarias del sistema financiero peruano durante el periodo 2012 al 2018; la volatilidad del tipo de cambio afecta a su vez significativamente a la dolarización de créditos y dolarización de depósitos.
\end{abstract}

Palabras clave: Volatilidad del tipo de cambio; dolarización; dolarización de créditos; dolarización de depósitos.

\begin{abstract}
The dollarization of the banking companies of the financial system have strong implications in the development and decision making of these to generate "mismatches" both currencies and terms, having incidence in the administration, planning, cost management, risk and others that finally affects the economic financial results of these companies. There are many causes that explain dollarization in banking companies. According to the seen in many countries, mainly developing countries and small economies, the volatility of the exchange rate has played an important role in the behavior of dollarization. The main results of the study are: the volatility of the exchange rate significantly influences the dollarization of the banking companies of the Peruvian financial system during the period 2012-2018; In turn, it significantly affects the dollarization of credits and dollarization of deposits.
\end{abstract}

Keywords: Volatility of the exchange rate; dollarization; dollarization of credits; dollarization of deposits.

\section{Emerson Yauri Lozano}

emerson.yauri@unmsm.edu.pe

Universidad Nacional Mayor de San Marcos, Facultad de Ciencias Administrativas. Lima, Perú

Presentado: 13/03/2020 - Aceptado: 02/10/2020 - Publicado: 26/11/2020

(C) Los autores. Este artículo es publicado por Gestión en el Tercer Milenio de la Facultad de Ciencias Administrativas de la Universidad Nacional Mayor de San Marcos. Este es un artículo de acceso abierto, distribuido bajo los términos de la licencia Creative Commons Atribucion - No Comercia_Compartir Igual 4.0 Internacional. (http://creativecommons.org/licenses/by-nc-sa/4.0/) que permite el uso no comercial, distribución y reproducción en cualquier medio, siempre que la obra original sea debidamente citada. 


\section{INTRODUCCIÓN}

En el estudio se quiere conocer la influencia de la volatilidad del tipo de cambio en la dolarización de las empresas bancarias del sistema financiero peruano en el periodo 2012 - 2018. Los aspectos que se han querido abordar, es decir los problemas específicos que se pretenden estudiar son: a) el efecto de la volatilidad del tipo de cambio a la dolarización de créditos de las empresas bancarias del sistema financiero y b) el efecto de la volatilidad del tipo de cambio a la dolarización de depósitos de las empresas bancarias.

La dolarización en las empresas bancarias del sistema financiero, que comprende a la dolarización de depósitos y dolarización de créditos, genera "descalces" tanto de monedas como de plazos, además al ser transversal a los diferentes sectores de la economía afecta y/o beneficia a estas empresas a través de los canales monetario, real, financiero, externo y las posiciones de comprador/vendedor teniendo finalmente incidencia en las empresas bancarias.

Definimos en este contexto a la dolarización de depósitos como el ratio de depósitos en dólares respecto a los depósitos totales mientras que a la dolarización de créditos como el ratio de créditos en dólares respecto a los créditos totales.

La dolarización de las empresas bancarias puede ser explicada por diversas causas. En países de economías pequeñas y en vías de desarrollo, se ha estudiado que la volatilidad del tipo de cambio ha tenido incidencia en la dolarización ya que las variaciones del tipo de cambio afectan a la toma de decisiones de los agentes que depositan en los bancos en relación a la elección del tipo de moneda en el cual realizar los depósitos ya sea en moneda nacional o extranjera (dólares) y por el lado de los bancos en qué moneda ofrecer créditos siendo por ende importante estudiar las implicancias que tiene sobre la dolarización más aún si se trata de variaciones bruscas del tipo de cambio o también variaciones grandes en un determinado momento y variaciones más pequeñas en otro. En el estudio definimos a la volatilidad del tipo de cambio como la desviación estándar del tipo de cambio.
El desarrollo del estudio se realiza en base al sustento teórico en el que se apoya Arteta (2002), quien analiza las relaciones entre los regímenes cambiarios incluyendo la volatilidad del tipo de cambio con la dolarización de las empresas bancarias (bancos), además estudia las causas de las crisis financieras y los principales efectos de la liberalización financiera. Menciona que en la dolarización de los bancos se incluye a la dolarización de los depósitos bancarios y a la dolarización de los créditos que otorgan los bancos los cuales en general están muy extendidos en los países en desarrollo lo que ocasiona diversos efectos a nivel de bancos y la economía en general ya que al realizarse la intermediación financiera se puede acentuar los efectos de la hoja de balance y con ello la fragilidad del sistema bancario.

Por otra parte, se tienen antecedentes del estudio en relación a esta investigación tanto nacional como internacional. Dentro de la primera se tiene a Quiroz (2008) con el estudio Función de reacción del BCRP y las fluctuaciones del tipo de cambio; Palomino (2013) quien realizó Análisis de los depósitos y colocaciones en la economía peruana por principales ciudades: 1995-2009; Jaramillo (2015) realiza Modelando la dinámica del tipo de cambio en el Perú, entre otros autores. En internacionales tenemos a Jaque (2007) con La dolarización y el riesgo cambiario en la banca múltiple; Pena (2008) con El riesgo tipo de cambio de instituciones financieras en economías dolarizadas y a Arguello (2007) quien realiza el estudio Dolarización y su impacto en las exportaciones y tasa de interés; entre otros autores.

\section{MÉTODOS}

El presente estudio es de tipo cuantitativo y correlacional ya que las variables incluidas en la investigación son de naturaleza cuantitativa. El diseño de investigación realizado es documental, ya que se realizó en base a información proporcionada de la Superintendencia de Banca, Seguros y AFP (SBS) y el Banco Central de Reserva del Perú (BCRP) respecto a tipo de cambio, depósitos en soles, depósitos en dólares, créditos en soles, créditos en dólares de los bancos de manera mensual durante el periodo 2012 a 2018. La unidad de análisis fue la empresa bancaria (banco) del sistema 
financiero peruano. La población estuvo conformada por el conjunto o total de empresas bancarias (bancos) del sistema financiero peruano que en total son 16 bancos, como en la población es reducido el número de unidades que la conforman y los datos secundarios necesarios para la realización de la investigación fueron accesibles en su totalidad, entonces se estudió a la población en su conjunto que es el número total de bancos (16).

En cuanto al análisis e interpretación de la información, los datos obtenidos de la revisión documentaria fueron procesados en el software estadístico SPSS. Posteriormente se hizo uso de estadísticas descriptivas para comprender a los datos, se desarrolló el análisis de correlaciones y finalmente se realizaron pruebas estadísticas de análisis multivariado para probar las hipótesis de investigación. El análisis incorpora implícitamente a los efectos de la puesta en marcha de la política de intervención cambiaria y de la política de desdolarización de créditos a partir del 2013 del BCRP basado en medidas de encaje adicional en función a límites al saldo de crédito en moneda extranjera, tanto para el caso de crédito total como del crédito hipotecario y vehicular ya que los datos utilizados en el análisis ya toman en cuenta a dichas políticas siendo también producto de ellas.

\section{RESULTADOS}

En la Tabla 1, se observa que en general existe correlación negativa significativa al 10\% de -0.048 entre la dolarización de créditos y la volatilidad del tipo de cambio dado el conjunto total de observaciones de los 16 bancos de forma mensual durante el periodo 2012 a 2018, esto puede explicarse debido al problema de descalce al que se enfrentan los que toman los créditos en dólares y los bancos que ofrecen los créditos, además de la política de desdolarización de créditos del BCRP.

En la Tabla 2, se muestra que la correlación de la dolarización de depósitos y la volatilidad del tipo de cambio es positiva y significativa de 0.051 a un nivel de 0.1 para el total de observaciones de los 16 bancos, explicado por los riesgos que trae el tipo de cambio incierto en los ingresos en soles futuros a partir de los ingresos generados en dólares de los depósitos.

En la Tabla 3, se observa que la correlación entre la dolarización de créditos y la dolarización de depósitos es significativa a un nivel de 0.01 y alta (valor de 0.923 ) para el total de observaciones, ello es explicado principalmente por las políticas de calce de los créditos con los depósitos en dólares por parte de los bancos.

Tabla 1

Correlación de la dolarización de créditos con la volatilidad del tipo de cambio

\begin{tabular}{llcc}
\multirow{2}{*}{ Dolarización de créditos } & \multicolumn{1}{c}{ Correlación de Pearson } & 1 & \multicolumn{1}{c}{ Volatilidad de tipo de cambio } \\
\cline { 2 - 4 } & Sig. (bilateral) & &,$- 048^{* *}$ \\
\hline \multirow{2}{*}{ Volatilidad de tipo de cambio } & Correlación de Pearson &,- 048 & \multicolumn{1}{c}{078} \\
\cline { 2 - 4 } & Sig. (bilateral) &, 078 & 1 \\
\hline
\end{tabular}

Nota: ** la correlación es significativa en el nivel 0.1. Elaboración propia.

Tabla 2

Correlación de la dolarización de depósitos con la volatilidad de tipo de cambio

\begin{tabular}{llcc}
\multirow{2}{*}{ Dolarización de depósitos } & \multicolumn{1}{c}{ Dolarización de depósitos } & \multicolumn{1}{c}{ Volatilidad de tipo de cambio } \\
\cline { 2 - 4 } & Correlación de Pearson & 1 &, $051^{* *}$ \\
\hline \multirow{2}{*}{ Volatilidad de tipo de cambion } & Correlación de Pearson &, 051 &, 062 \\
\cline { 2 - 4 } & Sig. (bilateral) &, 062 & 1 \\
\hline
\end{tabular}

Nota: ** la correlación es significativa en el nivel 0.1. Elaboración propia. 
En la Figura 1, se muestra un análisis de dispersión entre dolarización de créditos y volatilidad del tipo de cambio, se observa una relación inversa (negativa) debido a cambios en la riqueza de los agentes al variar el tipo de cambio.

Por otra parte, en la Figura 2, se muestra un análisis de dispersión entre la dolarización de depósitos y la volatilidad del tipo de cambio también se observa una relación directa (positiva) entre la dolarización de depósitos y la volatilidad del tipo de cambio debido a cambios en los ingresos en soles de los depositantes en dólares.

Por otro lado, en la Tabla 4, al realizarse las pruebas de análisis multivariado se observa que la volatilidad del tipo de cambio influye significativamente sobre la dolarización al 1\% de significancia, donde se muestran 4 estadísticos de prueba de significancia, en todos los estadísticos se muestra que la influencia es significativa, generalmente el estadístico de Lambda de Wilks es el más usado. Ello dado que como se muestra en las Tablas 5 y 6, la volatilidad del tipo de cambio afecta tanto a la dolarización de créditos y a la dolarización de depósitos las cuales son dimensiones de la dolarización.

En la Tabla 5, se observa que la volatilidad del tipo de cambio afecta significativamente sobre la dolarización de créditos al 1\% de significancia, acorde al estadístico F y del Tipo III de suma de cuadrados utilizado por SPSS por defecto en el análisis multivariado.

Ello se debe a que las empresas y familias cuando se endeudan en dólares y los ingresos que perciben son en soles y se dan variaciones en el tipo de cambio, su riqueza varía teniendo problemas de liquidez.

Tabla 3

Correlación de la dolarización de créditos con la dolarización de depósitos

\begin{tabular}{llcc}
\multirow{2}{*}{ Dolarización de créditos } & \multicolumn{2}{c}{ Dolarización de créditos } & \multicolumn{2}{c}{ Dolarización de depósitos } \\
\cline { 2 - 3 } & Correlación de Pearson & 1 &, $923^{* *}$ \\
\hline \multirow{2}{*}{ Dolarización de depósitos } & Sigilateral) & &, 000 \\
\cline { 2 - 3 } & Correlación de Pearson &, $923^{* *}$ & 1 \\
\hline
\end{tabular}

Nota: ** La correlación es significativa en el nivel 0,01 (2 colas). Elaboración propia.

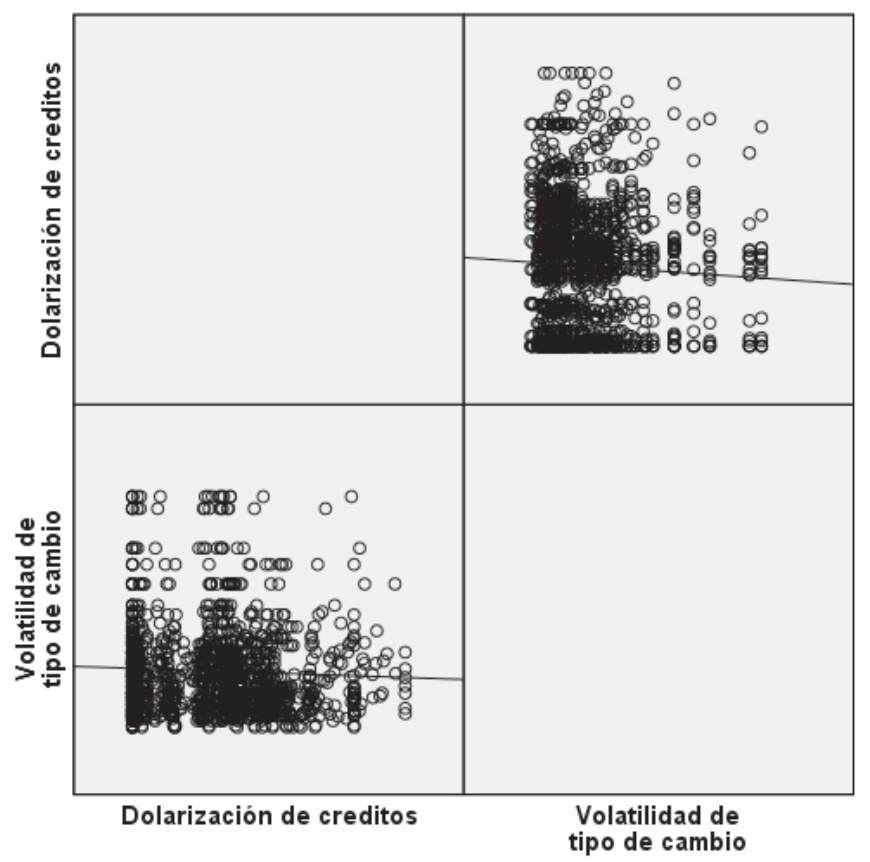

Figura 1. Dispersión de la dolarización de créditos con la volatilidad del tipo de cambio. 
Volatilidad de tipo de cambio y dolarización de empresas bancarias del sistema financiero peruano, 2012 - 2018



Figura 2. Dispersión de la dolarización de depósitos con la volatilidad del tipo de cambio.

Elaboración propia.

Tabla 4

Análisis multivariado de la influencia de la volatilidad del tipo de cambio sobre la dolarización

\begin{tabular}{llcccccc} 
Efecto & Valor & F & Gl de hipótesis & gl de error & Sig. \\
& Traza de Pillai &, 079 & $56,632^{\mathrm{b}}$ & 2,000 & 1326,000 &, 000 \\
\cline { 2 - 8 } $\begin{array}{l}\text { Volatilidad de } \\
\text { tipo de cambio }\end{array}$ & Lambda de Wilks &, 921 & $56,632^{\mathrm{b}}$ & 2,000 & 1326,000 &, 000 &, 000 \\
\cline { 2 - 8 } & Traza de Hotelling &, 085 & $56,632^{\mathrm{b}}$ & 2,000 & 1326,000 &, 000 \\
\cline { 2 - 8 } & Raíz mayor de Roy &, 085 & $56,632^{\mathrm{b}}$ & 2,000 & 1326,000 &, \\
\hline
\end{tabular}

Nota: Elaboración propia.

Tabla 5

Análisis multivariado del efecto de la volatilidad del tipo de cambio sobre la dolarización de créditos

\begin{tabular}{llcccccc} 
Origen & Variable dependiente & $\begin{array}{c}\text { Tipo III de suma } \\
\text { de cuadrados }\end{array}$ & gl & $\begin{array}{c}\text { Cuadrático } \\
\text { promedio }\end{array}$ & F & Sig. \\
$\begin{array}{l}\text { Volatilidad de } \\
\text { tipo de cambio }\end{array}$ & Dolarización de créditos &, 176 & 1 &, 176 & 40,492 &, 000 \\
\cline { 2 - 8 } & Dolarización de depósitos &, 203 & 1 &, 203 & 58,579 &, 000 \\
\hline
\end{tabular}

Nota: Elaboración propia.

Tabla 6

Análisis multivariado del efecto de la volatilidad del tipo de cambio sobre la dolarización de depósitos

\begin{tabular}{llccccc} 
Origen & Variable dependiente & $\begin{array}{c}\text { Tipo III de suma } \\
\text { de cuadrados }\end{array}$ & gl & $\begin{array}{c}\text { Cuadrático } \\
\text { promedio }\end{array}$ & F & Sig. \\
\cline { 2 - 8 } $\begin{array}{l}\text { Volatilidad de } \\
\text { tipo de cambio }\end{array}$ & Dolarización de créditos &, 176 & 1 &, 176 & 40,492 &, 000 \\
\cline { 2 - 9 } & Dolarización de depósitos &, 203 & 1 &, 203 & 58,579 &, 000 \\
\hline
\end{tabular}

Nota: Elaboración propia. 
Finalmente, en la Tabla 6 se muestra que la volatilidad del tipo de cambio afecta significativamente sobre la dolarización de depósitos al $1 \%$ de significancia teniendo en cuenta el estadístico F y del Tipo III de suma de cuadrados.

Esto es explicado por los efectos generados en los ingresos en soles de los depositantes en dólares generándose riesgos ante movimientos en el tipo de cambio.

\section{DISCUSIÓN}

Se comprueba lo mencionado por los enfoques teóricos que consideran que la volatilidad del tipo de cambio es uno de los determinantes e influye sobre la dolarización de las empresas bancarias del sistema financiero.

Respecto a las dimensiones de la dolarización como son: la dolarización de créditos y la dolarización de depósitos; se corrobora lo mencionado por Arteta (2002), en el sentido que la volatilidad del tipo de cambio influye tanto a la dolarización de créditos como a la dolarización de depósitos de las empresas bancarias.

Por otra parte, en relación a los antecedentes mencionados en la investigación, se corrobora lo mencionado por Palomino (2013) ya que en un contexto de volatilidad del tipo de cambio existen cambios en la dolarización de empresas bancarias ya que existen variaciones en el ratio de depósitos y de colocaciones variando la dolarización de depósitos y de créditos.

En relación a Jaque (2007) también se corrobora con esta investigación lo mencionado en dicho artículo ya que una mayor volatilidad del tipo de cambio influye generando vulnerabilidades en la dolarización de la banca múltiple tanto a nivel de depósitos como de créditos.

Respecto al trabajo de Pena (2008) quien afirma que el riesgo de tipo de cambio genera más vulnerabilidades a los accionistas y depositantes, el presente trabajo guarda concordancia con ello ya que acorde a éste la volatilidad del tipo de cambio afecta a la dolarización y particularmente a la dolarización de depósitos.

\section{CONCLUSIONES}

Se concluye que la volatilidad del tipo de cambio influye significativamente en la dolarización de las empresas bancarias del sistema financiero peruano durante el periodo 2012 2018 al 1\% de significancia.

Por otra parte, también concluimos que la volatilidad del tipo de cambio afecta significativamente a la dolarización de créditos al $1 \%$ de significancia debido al problema de descalce de créditos principalmente.

Además, se concluye que la volatilidad del tipo de cambio afecta significativamente a la dolarización de depósitos al 1\% de significancia debido al riesgo de los retornos en soles de los depositantes en dólares ante variaciones del tipo de cambio.

\section{REFERENCIAS BIBLIOGRÁFICAS}

Arguello, C. (2007). Dolarización y su impacto en las exportaciones y tasa de interés (Tesis de Titulación). Pontificia Universidad Católica de Chile, Chile.

Arteta, C. (2002). Exchange Rate Regimes and Financial Dollarization: Does Flexibility Reduce Bank Currency Mismatches? Federal Reserve Board International Finance, Working Paper $\mathrm{N}^{\circ} 738$.

Jaque, R. (2007). La dolarización y el riesgo cambiario en la banca múltiple, Secretaría de Estado de Economía, Planificación y Desarrollo, República Dominicana.

Jaramillo, M. (2015). Modelando la dinámica del tipo de cambio en el Perú, Superintendencia de Banca, Seguros y AFPs, Perú.

Palomino, M. (2013). Análisis de los depósitos y colocaciones en la economía peruana por principales ciudades: 1995-2009, Universidad San Martín de Porres, Perú.

Pena, A. (2008). El riesgo tipo de cambio de instituciones financieras en economías dolarizadas, Superintendencia de Instituciones de Intermediación Financiera (SIIF), Uruguay.

Quiroz, O. (2008). Función de reacción del BCRP y las fluctuaciones del tipo de cambio. Pontificia Universidad Católica del Perú, Perú. 